\title{
THE MAXIMAL QUOTIENT RINGS OF REGULAR GROUP RINGS III
}

\author{
Ferran Cedó
}

To the memory of Andreu Pitarch

Abstract

We give a new proof of the main result of [1] which does not use the classification of the finite simple groups.

Introduction. In [1] the proof of the main result, [1, Theorem 2.3], is based implicitly on the classification of the finite simple groups through the use of [6, Theorem]. We give here a proof of [1, Theorem 2.3] which does not use $[\mathbf{6}$, Theorem].

The proof. Let $G$ be a group. Recall that $\Delta(G)=\{g \in G \mid[G$ : $\left.\left.C_{G}(g)\right]<\infty\right\}$ is a characteristic subgroup of $G$. A group $G$ is an FC-group (finite conjugate group) if $G=\Delta(G)$. A group $G$ satisfies Min (minimal condition on subgroups) if every non-empty set of subgroups of $G$, partially ordered by inclusion, has a minimal element. A group satisfies Min- $p$ for the prime $p$ if each of its $p$-subgroups satisfies Min.

Throughout, $K$ denotes a field and $G$ a locally finite group with no elements of order $\operatorname{char}(K)$. Thus $K[G]$ is a regular group ring (cf. [9, Theorem 3.1.5]).

Lemma 1. Suppose that $G$ is an FC-group satisfying Min-p for all primes $p$ and that the type $I_{f}$ part of $Q^{r}(K[G])$ is non-zero. Then $G$ is abelian-by-finite.

Proof: Suppose that $G$ has no abelian subgroup of finite index. By $[\mathbf{9}$, Lemma 6.3.3] and [1, Lemma 1.3(ii)], we may assume that $G$ is countable. By [10, Lemma 6] and [1, Lemma $1.3($ ii)], we may assume

$$
G=\left(\prod_{i=1}^{\infty} H_{i}\right) / H,
$$


where $\prod_{i=1}^{\infty} H_{i}$ is a (weak) direct product of finite groups $H_{i}$. We may also assume that $H \cap H_{i}=\langle 1\rangle$ for all $i$. Since $H$ and $H_{i}$ are disjoint normal subgroups of $\prod_{i=1}^{\infty} H_{i}$, they commute and we have

$$
H \leq Z\left(\prod_{i=1}^{\infty} H_{i}\right)=\prod_{i=1}^{\infty} Z\left(H_{i}\right)
$$

By [1, Lemmas 1.3(ii), 1.4 and Proposition 1.1], we may assume that the $H_{i}$ are not nilpotent and every proper subgroup of $H_{i}$ is abelian.

By [8], $H_{i}=P_{i}\left\langle t_{i}\right\rangle$, where $P_{i}$ is a normal Sylow $p_{i}$-subgroup of $H_{i}$ and $t_{i} \in H_{i}$ is an element of order $q_{i}^{n_{i}}\left(n_{i} \geq 1\right)$, where $q_{i}$ is a prime. Furthermore, $\left\langle t_{i}\right\rangle$ is not normal in $H_{i}$ and $\left\langle t_{i}^{q_{i}}\right\rangle=Z\left(H_{i}\right)$.

Since the type $I_{f}$ part of $Q^{r}(K[G])$ is non-zero, by [3, Lemma 4.2], there exists a non-zero abelian idempotent $e \in K[G]$. Thus there exists an integer $n$, such that

$$
\langle\operatorname{Supp} e\rangle \leq\left(\prod_{i=1}^{n} H_{i}\right) H / H \leq G
$$

Since $G$ satisfies Min- $p$ for all primes $p$, it is easy to see that there are infinitely many distinct $q_{i}$. Let $q_{j}$ be such that $j>n$ and $\langle\operatorname{Supp} e\rangle$ has no element of order $q_{j}$. Let $\tilde{t}_{j}=1+t_{j}+\cdots+t_{j}^{q_{j}^{n_{j}}-1}$. Note that $\tilde{t}_{j} / q_{j}^{n_{j}}$ is idempotent. Since $\left(x, t_{j}\right)=1$ for all $x \in \operatorname{Supp} e, e \tilde{t}_{j} / q_{j}^{n_{j}} \in e K[G] e$ is idempotent. Since $e$ is abelian and $(x, h)=1$ for all $x \in \operatorname{Supp} e$ and $h \in H_{j}$, we have

$$
e \tilde{t}_{j} h=e h \tilde{t}_{j}
$$

for all $h \in H_{j}$. Using the fact that $\sum_{i=0}^{q_{j}^{n_{j}}-1} K[\langle\operatorname{Supp} e\rangle] t_{j}^{i}$ is a direct sum, we deduce that $x t_{j} \in \operatorname{Supp} e \tilde{t}_{j}$ for all $x \in \operatorname{Supp} e$. Thus, given $x \in \operatorname{Supp} e$ and $h \in H_{j}$, there exist $y \in \operatorname{Supp} e$ and $m$ such that $x t_{j} h=y h t_{j}^{m}$. Hence $t_{j} h t_{j}^{-m} h^{-1}=x^{-1} y \in\langle\operatorname{Supp} e\rangle \cap H_{j}=\langle\operatorname{Supp} e\rangle \cap Z\left(H_{j}\right)$. Since $Z\left(H_{j}\right)$ is a $q_{j}$-group and $\langle\operatorname{Supp} e\rangle$ has no element of order $q_{j}$, we see $h^{-1} t_{j} h=t_{j}^{m}$. But $\left\langle t_{j}\right\rangle$ is not normal in $H_{j}$, which is a contradiction, so the lemma is proved.

Let $J$ be a right ideal of $K[G]$. As in [3], we define $\alpha(J, F)=\operatorname{dim}(J \cap$ $K[F]) /|F|$ for each finite subgroup $F$ of $G$, and $\alpha(J)=\sup \alpha(J, F)$, where $F$ ranges over all finite subgroups of $G$.

We denote by $\pi(G)$ the set of all primes $p$ such that $G$ has an element of order $p$. If $\pi$ is a set of primes we say that $G$ is a $\pi$-group if $\pi(G) \subseteq \pi$. 
Lemma 2. Suppose that $G$ satisfies Min-p for all primes $p$ and that the type $I_{f}$ part of $Q^{r}(K[G])$ is non-zero. Then $G$ is abelian-by-finite.

Proof: By [9, Lemma 6.3.3] and [1, Lemma 1.3(ii)], we may assume that $G$ is countable. By $[\mathbf{1}$, Lemma $1.3(\mathrm{ii})]$ and Lemma $1, \Delta(G)$ is abelian-by-finite. By [9, Lemma 12.1.2], $\Delta(G)$ has a characteristic abelian subgroup $A$ of finite index. Let $\sigma=\pi(\Delta(G) / A)$.

Since the type $I_{f}$ part of $Q^{r}(K[G])$ is non-zero, by [3, Lemma 4.2], there exists a non-zero abelian idempotent $e \in K[G]$. Let $H=\langle\operatorname{Supp} e\rangle$ and $\tau=\pi(H)$. Hence $\pi=\sigma \cup \tau$ is a finite set of primes.

Now $A$ is the direct product $A=\prod A_{p}$ of its $p$-primary parts. Let $A_{\pi^{\prime}}=\prod_{p \notin \pi} A_{p}$. Then $A_{\pi^{\prime}}$ is characteristic in $G$. Consider $\bar{G}=G / A_{\pi^{\prime}}$. Let $\delta \in K[G]$. We denote by $\bar{\delta}$ the image of $\delta$ in $K[\bar{G}]$. By [4, Lemma 7.6], $\bar{e}$ is an abelian idempotent, and clearly it is non-zero. Let $p$ be a prime such that $\alpha(\bar{e} K[\bar{G}])>p^{-1}$ and $p \notin \pi$. We shall see that $p \notin \pi(\bar{G})$.

Suppose that $p \in \pi(\bar{G})$. Then there exists $g \in G \backslash \Delta(G)$ with $o(g)=p^{n}$ and $o(\bar{g})=p$. Let $\tilde{g}=1+g+\cdots+g^{p^{n}-1}$. Since $K[G]$ is regular, there exists $\beta \in K[G]$ such that

$$
\tilde{g} e=\tilde{g} e \beta \tilde{g} e .
$$

By squaring it, we see that $e \beta \tilde{g} e$ is an idempotent in $e K[G] e$. By $[\mathbf{2}$, Lemma 2.1], Suppe $e \tilde{g} e \subseteq \Delta(G) H$. Let $H_{1}=\langle\operatorname{Supp} e \beta \tilde{g} e \cup \operatorname{Supp} e\rangle$. Thus $p \notin \pi\left(\bar{H}_{1}\right)$. Using the fact that we have a direct sum $\sum_{i=0}^{p-1} \bar{g}^{i} K\left[\bar{H}_{1}\right]$, we deduce that

$$
p^{n-1} \bar{e}=p^{n-1} \overline{e \beta \tilde{g} e} .
$$

Since $p \neq 0$ in $K, \bar{e}=\overline{e \beta \tilde{g} e}$. Thus $\bar{e} K[\bar{G}] \cong \overline{\tilde{g} e \beta} K[\bar{G}]$. By $[\mathbf{3}$, Lemma 1.2(iv)], $\alpha(\bar{e} K[\bar{G}]) \leq \alpha(\overline{\tilde{g}} K[\bar{G}])$. But an easy calculation shows that $\alpha(\overline{\tilde{g}} K[\bar{G}])=p^{-1}$. This contradicts the choice of $p$. Thus $p \notin \pi(\bar{G})$. Hence $\pi(\bar{G})$ is finite.

Since $\bar{e}$ is a non-zero abelian idempotent, by [3, Lemma 4.2], the type $I$ part of $Q^{r}(K[\bar{G}])$ is non-zero. By $[\mathbf{1}$, Proposition 1.2$],[\bar{G}: \Delta(\bar{G})]<\infty$ and $\Delta(\bar{G})^{\prime}$ is finite. By $[\mathbf{7}$, Theorem 3.13], $\Delta(\bar{G})$ satisfies Min- $p$ for all primes $p$. By $[\mathbf{1}$, Lemma 1.4], $[\Delta(\bar{G}): Z(\Delta(\bar{G}))]<\infty$. Thus $\bar{G}$ is abelian-by-finite. By $[\mathbf{1}$, Lemma $1.3($ ii) $]$, we may assume that $\bar{G}$ is abelian.

Let $\pi_{1}=\pi \cup \pi(\bar{G})$ and let $A_{\pi_{1}^{\prime}}=\prod_{p \notin \pi_{1}} A_{p}$. Then $\pi\left(G / A_{\pi_{1}^{\prime}}\right) \subseteq \pi_{1}$. By [9, Lemma 12.4.12], there exists a $\pi_{1}$-subgroup $Q$ of $G$ with $G=A_{\pi_{1}^{\prime}} Q$. By [1, Lemma 1.3(ii), Proposition 1.2 and Lemma 1.4], $Q$ is abelianby-finite. By [1, Lemma 1.3(ii)], we may assume that $Q$ is abelian. Since $\pi(Q)$ is finite and satisfies Min- $p$ for all primes $p, Q$ has a minimal 
subgroup of finite index. Thus by [1, Lemma $1.3($ ii) $]$, we may assume that $Q$ contains no proper subgroup of finite index.

We shall see that $G$ is abelian. Let $q$ be a prime such that $q \notin \pi_{1}$. By [1, Lemma 1.3(ii), Proposition 1.2 and Lemma 1.4], $A_{q} Q$ has an abelian normal subgroup $B$ of finite index. Now $[Q: Q \cap B]<\infty$, thus $Q \leq B$. Now $B$ is the direct product $B=\prod B_{p}$ of its $p$-primary parts. Let $B_{\pi_{1}}=\prod_{p \in \pi_{1}} B_{p}$. Since $Q$ is a $\pi_{1}$-group, $Q \leq B_{\pi_{1}}$. Thus $A_{q} Q=A_{q} B_{\pi_{1}}$. Since $B_{\pi_{1}}$ is a normal subgroup of $A_{q} B_{\pi_{1}}, A_{q} Q$ is abelian. Hence $G=A_{\pi_{1}^{\prime}} Q$ is abelian.

Theorem 3 ([1, Theorem 2.3]). The type $I_{f}$ part of $Q^{r}(K[G])$ is non-zero iff $[G: \Delta(G)]<\infty$ and $\Delta(G)^{\prime}$ is finite. Furthermore, in this case the type $I_{f}$ part of $Q^{r}(K[G])$ is isomorphic to $Q^{r}(K[G / M])$, where $M=\cap L^{\prime}$ and the intersection is over all subgroups $L$ of $G$ of finite index.

Proof: The proof of the "if" part and the second part is as in [1].

Suppose that the type $I_{f}$ part of $Q^{r}(K[G])$ is non-zero. Suppose that $[G: \Delta(G)]=\infty$ or $\left|\Delta(G)^{\prime}\right|=\infty$. By [1, Lemma 1.3(i)], there exists a non-zero central idempotent $u \in K[G]$ such that $u K[G]$ has bounded index of nilpotence. Thus, by $[\mathbf{9}$, Theorem 5.3.15], $u K[G]$ does not satisfy any polynomial identity. By $[\mathbf{1}$, Lemma 2.2$]$, there exists an irreducible $u K[G]$-module $V$ with representation $\rho: K[G] \rightarrow$ End $V$ such that $\rho(G)$ has no abelian subgroup of finite index.

Since $u K[G]$ has bounded index of nilpotence, by [4, Corollary 7.10], $V$ is finite dimensional over its commuting ring. By [5, Lemma 2.4], $\rho(G)$ satisfies Min- $p$ for all primes $p$. Let $\varphi: K[G] \rightarrow K[\rho(G)]$ the natural projection. It is easy to see that $\varphi(u)$ is a non-zero central idempotent of $K[\rho(G)]$. By [4, Proposition 7.7], $\varphi(u) K[\rho(G)]$ has bounded index of nilpotence. By [4, Corollary 7.4 and Theorems 7.20 and 10.24], the type $I_{f}$ part of $Q^{r}(K[\rho(G)])$ is non-zero. By Lemma $2, \rho(G)$ is abelian-byfinite, a contradiction, thus $[G: \Delta(G)]<\infty$ and $\left|\Delta(G)^{\prime}\right|<\infty$.

Acknowledgements. This work was partially supported by the DGICYT through grant PB92-0586.

\section{References}

1. F. CEDó, On the maximal quotient ring of regular group rings, $J$. Algebra 115 (1988), 164-174.

2. F. CEDó, The maximal quotient ring of regular group rings. II, Proc. Amer. Math. Soc. 104 (1988), 357-362. 
3. F. Cedó And B. Hartley, Maximal quotient rings and essential right ideals in group rings of locally finite groups, Publ. Mat. 36 (1992), 473-480.

4. K. R. Goodearl, "Von Neumann Regular Rings," Pitman, London, 1979.

5. B. Hartley, Injective modules over group rings, Quart. J. Math. Oxford Ser. (2) 28 (1977), 1-29.

6. B. Hartley and M. A. Shahabi Shojaei, Finite groups of matrices over division rings, Math. Proc. Cambridge Philos. Soc. 92 (1982), 55-64.

7. O. H. Kegel And B. A. F. Wehrfritz, "Locally Finite Groups," North-Holland, Amsterdam, London, 1973.

8. G. A. Miller And H. Moreno, Nonabelian groups in which every subgroup is abelian, Trans. Amer. Math. Soc. 4 (1903), 398-404.

9. D. S. Passman, "The Algebraic Structure of Group Rings," WileyInterscience, New York, 1977.

10. R. L. Snider, Group algebras whose simple modules are finite dimensional over their commuting rings, Comm. Algebra 2 (1974), 15-25.

Departament de Matemàtiques

Universitat Autònoma de Barcelona

08193 Bellaterra (Barcelona)

SPAIN

Rebut el 16 de Juny de 1994 